\title{
A multi-stage procedure of Bayesian estimation for first order moving average model
}

\author{
Mahmoud M. Smadi ${ }^{1}$, Amal A. Al-Husban ${ }^{2}$ \\ ${ }^{I}$ Department of Mathematics and Statistics Jordan University of Science and Technology \\ P.O.BOX 3030, Irbid 22110 Jordan \\ ${ }^{2}$ Ministry of Education, Jordan \\ *Corresponding author E-mail: smadi@just.edu.jo
}

\begin{abstract}
In this paper we present a multi-stage procedure for improving the Bayes estimates of first order moving average model (MA(1)) using the estimated residuals. Simulation results based on different model structures with varying numbers of observations are used to investigate the performance of the proposed procedure. The results show a remarkable improvement of the $t$-approximation using two and three stage procedure in terms of the posterior mean and variance for different model structures. It is also noted that these estimates converge as the series lengths increase.
\end{abstract}

\section{Introduction}

The Bayesian inferences on autoregressive moving average (ARMA) models are limited due to the complicated form of the likelihood function which makes it difficult to get tractable marginal posteriors and Bayes estimates. Shaarawy and Bromeling, [1] proposed an approximate Bayes estimates based on the estimated residuals for the analysis of MA and ARMA models. Using the estimated residuals, they obtain marginal posteriors, Bayes estimates, and predictive densities in a closed form. Asymptotic justification of the Bayes estimates of $\operatorname{MA}(q)$ model is given by Smadi and AbuTaleb [3].

The estimated residuals became a popular method in Bayesian inference of different parametric time series models. Cathy [6] used the estimated residuals in Bayesian inference of the bilinear models. Smadi [2] and Safadi and Morettin [5] have used this estimated residuals approach to explore the posterior distributions of the threshold autoregressive moving average models. Smadi [7] used the estimated residuals in Bayesian inference of threshold moving average models. On the other hand, Koreisha and Pukkila [4] use the estimated residuals by fitting long autoregression and developed a generalized least-squares approach for estimation of ARMA models.

In this work, we propose a multi-stage procedure for improving the Bayes estimates of first order moving average model (MA(1)) using the estimated residuals. Simulation results based on different model structures with varying numbers of observations are used to investigate the performance of the proposed procedure.

\section{Bayes estimation for MA(1) model}

The first order moving average model $(\mathrm{MA}(1))$ is given by:

$$
X_{t}=Z_{t}-\theta Z_{t-1} \quad t=0, \pm 1, \pm 2, \ldots,
$$

where $\left\{Z_{t}\right\}$ are i.i.d $N\left(0, \sigma^{2}\right)$ random variables.

Shaarawy and Bromeling (1984) derived approximate marginal posterior distributions and posterior means for the parameters of the $\operatorname{MA}(q)$ model. For $\operatorname{MA}(1)$ model, the true innovations $\left\{Z_{t}\right\}$ are first estimated as $\left\{\hat{Z}_{t}\right\}$ and calculated recursively using $\hat{Z}_{t}=X_{t}+\hat{\theta} \hat{Z}_{t-1} \quad(t=1, \ldots, n)$, where $\hat{Z}_{0}=0$, and $\hat{\theta}$ is the least squares estimate of $\theta$. They assume a multi-normal-gamma prior density on the model parameters of the $\operatorname{MA}(q)$ model. For the MA(1) model, the normal-gamma prior density on $\theta$ and $\tau$ reduces to 
$g(\theta, \tau)=\tau^{\alpha-1} e^{-\tau \beta} e^{-\frac{\tau p}{2}(\theta-\mu)^{2}}$, where $\theta \in R, \tau>0, \alpha>0, \beta>0, \mu \in R$

Based on Shaarawy and Bromeling

(1984) results, the following results follow:

(i) The marginal posterior density of $\theta$ has a $t$-distribution with $n+2 \alpha-1$ degrees of freedom. The mean and precision are given by

$E(\theta \mid \underline{X})=\frac{p \mu-\sum_{t=1}^{n} x_{t} \hat{z}_{t-1}}{p+\sum_{t=1}^{n} \hat{z}_{t-1}^{2}}$

Precision $=\frac{(n+2 \alpha) \sum_{t=1}^{n} \hat{z}_{t-1}^{2}}{\mu^{2} p+2 \beta+\sum_{t=1}^{n} x_{t}^{2}-\left(\sum_{t=1}^{n} x_{t} \hat{z}_{t-1}\right)^{2} / \sum_{t=1}^{n} z_{t-1}^{2}}$

The marginal posterior density of $\tau$ is a gamma distribution, where;

$\alpha^{\prime}=\frac{n+2 \alpha}{2}$

and;

$2 \beta^{\prime}=\mu^{2} p+2 \beta+\sum_{t=1}^{n} x_{t}^{2}-\left(\sum_{t=1}^{n} x_{t} \hat{z}_{t-1}\right)^{2} / \sum_{t=1}^{n} \hat{z}_{t-1}^{2}$

\section{Bayes estimation using multi-stage procedure}

The above Bayes estimates depends heavily on the estimated residuals, since the estimated residuals are estimates of the true innovations, they may contain random variation due to estimation errors, and consequently, may yield inefficient parameter estimates. The simulation results below shows poor performance of such estimates especially for small and moderate series lengths.

In the following a proposed multi-stage procedure for improving the above $t$-approximation is given as follows:

Step 1: Find the LSE of $\theta$.

Step 2: Calculate the estimated residuals recursively;

$, \hat{Z}_{0}=0, \hat{Z}_{t}=X_{t}+\hat{\theta} \hat{Z}_{t-1}(t=1, \ldots, n)$

Step 3: Calculate the Bayes estimate which is the mean of the $t$-distribution using

$E(\theta \mid \underline{X})=\frac{p \mu-\sum_{t=1}^{n} x_{t} \hat{z}_{t-1}}{p+\sum_{t=1}^{n} \hat{z}_{t-1}^{2}}$

Step 4: Use the Bayes estimate is step 3 to estimate again the residuals $\left\{\hat{Z}_{t}\right\}$ recursively as in step 2.

Step 5: Calculate the Bayes estimate given in step 3 using the updated estimated residuals computed in step 4.

Step 6: Repeat the above procedure $k$-steps.

\section{Simulation results and discussion}

A simulation study to investigate the performance of the proposed procedure was performed. Different parameter values are used, $\theta=-0.8,0.8$ step $0.2, \sigma^{2}=1$ with series $n=50,100,200$, and 300 are used. Estimates based on two and 3 steps are computed and compared with Bayes estimate based on one step.

The following subroutines from the IMSL statistical library were used to obtain least squares estimates:

1. LSLSE which compute preliminary estimates of the autoregressive and moving average parameters of an ARMA model.

2. NLLSE which computes least-squares estimates of parameters for ARMA model.

One thousand realizations are used in the simulation study. The posterior means and variances were computed using one, two, and three stage procedure. The results are given in tables (1), (2), (3), and (4). 
The results in tables (1) to (4) show poor performance of $t$-approximation using one stage, especially for $\theta>0.5$ and $\theta<-$ 0.5 . It is noted a remarkable improvement of the t-approximation using two and three stage procedure in terms of the posterior mean and variance for different model structures, especially near the invertibility boundaries -1 and 1 . It is also noted that the estimates based on two and three stage procedures converges as the series lengths increases.

Generalization of the proposed multi-stage procedure to general MA, ARMA, and other parametric time series models like Bilinear and threshold MA and ARMA models is straight forward.

Table 1: The $t$-approximation, $n=50$

\begin{tabular}{lllllll}
\hline & One step & \multicolumn{3}{c}{ Three step } \\
\cline { 2 - 7 }$\theta$ & $\mathrm{E}(\theta \mid \mathrm{x})$ & $\mathrm{V}(\theta \mid \mathrm{x})$ & $\mathrm{E}(\theta \mid \mathrm{x})$ & $\mathrm{V}(\theta \mid \mathrm{x})$ & $\mathrm{E}(\theta \mid \mathrm{x})$ & $\mathrm{V}(\theta \mid \mathrm{x})$ \\
\hline-0.8 & -.6116 & .0882 & -.7076 & .0349 & -.7495 & .0263 \\
-0.6 & -.4922 & .0466 & -.5756 & .0201 & -.5929 & .0228 \\
-0.4 & -.3760 & .0246 & -.3973 & .0214 & -.3984 & .0223 \\
-0.2 & -.2002 & .0227 & -.1999 & .0225 & -.1993 & .0225 \\
0.2 & .2040 & .0235 & .2004 & .0224 & .2011 & .0225 \\
0.4 & .4003 & .0226 & 3985. & .0213 & .4004 & .0226 \\
0.6 & .5748 & .0331 & 5861. & .0194 & .5961 & .0230 \\
0.8 & .7537 & .0458 & .7199 & .0355 & .7619 & .0237 \\
\hline
\end{tabular}

Table 2: The $t$-approximation, $n=100$

\begin{tabular}{|c|c|c|c|c|c|c|}
\hline \multirow[b]{2}{*}{$\theta$} & \multicolumn{2}{|c|}{ One step } & \multicolumn{2}{|c|}{ Two step } & \multicolumn{2}{|c|}{ Three step } \\
\hline & $\mathrm{E}(\theta \mid \mathrm{x})$ & $\mathrm{V}(\theta \mid \mathrm{x})$ & $\mathrm{E}(\theta \mid \mathrm{x})$ & $\mathrm{V}(\theta \mid \mathrm{x})$ & $\mathrm{E}(\theta \mid \mathrm{x})$ & $\mathrm{V}(\theta \mid \mathrm{x})$ \\
\hline-0.8 & -.6626 & .0619 & -.7444 & .0144 & -.7807 & .0119 \\
\hline-0.6 & -.5266 & .0354 & -.5833 & .0081 & -.5989 & .0099 \\
\hline-0.4 & -.3968 & .0108 & -.3992 & .0093 & -.3996 & .0097 \\
\hline-0.2 & -.2016 & .0097 & -.2000 & .0096 & -.1999 & .0096 \\
\hline 0.2 & .2019 & .0099 & .1999 & .0096 & .2001 & .0097 \\
\hline 0.4 & .4020 & .0102 & .3992 & .0095 & .3996 & .0097 \\
\hline 0.6 & .5700 & .0227 & .5901 & .0085 & .5988 & .0101 \\
\hline 0.8 & .7173 & .0563 & .7456 & .0164 & .7840 & .0113 \\
\hline
\end{tabular}

Table 3: The $t$-approximation, $n=200$

\begin{tabular}{|c|c|c|c|c|c|c|}
\hline \multirow[b]{2}{*}{$\theta$} & \multicolumn{2}{|c|}{ One step } & \multicolumn{2}{|c|}{ Two step } & \multicolumn{2}{|c|}{ Three step } \\
\hline & $\mathrm{E}(\theta \mid \mathrm{x})$ & $\mathrm{V}(\theta \mid \mathrm{x})$ & $\mathrm{E}(\theta \mid \mathrm{x})$ & $\mathrm{V}(\theta \mid \mathrm{x})$ & $\mathrm{E}(\theta \mid \mathrm{x})$ & $\mathrm{V}(\theta \mid \mathrm{x})$ \\
\hline-0.8 & -.6766 & .0659 & -.7505 & .0095 & -.7884 & .0053 \\
\hline-0.6 & -.5624 & .0220 & -.5911 & .0046 & -.5997 & .0050 \\
\hline-0.4 & -.4013 & .0052 & -.4002 & .0049 & 4003-. & .0050 \\
\hline-0.2 & -.2015 & .0050 & -.2005 & .0050 & $2005-$. & .0050 \\
\hline 0.2 & .2005 & .0050 & .1995 & .0050 & .1995 & .0050 \\
\hline 0.4 & .4010 & .0051 & .3992 & .0049 & .3985 & .0050 \\
\hline 0.6 & .5782 & .0160 & .5929 & .0047 & .5985 & .0051 \\
\hline 0.8 & 6934. & .0654 & .7515 & .0105 & .7882 & .0050 \\
\hline
\end{tabular}

Table 4: The $t$-approximation, $n=300$

\begin{tabular}{llllll}
\hline & One step & \multicolumn{3}{c}{ Three step } \\
\cline { 2 - 5 }$\theta$ & $\mathrm{E}(\theta \mid \mathrm{x})$ & $\mathrm{V}(\theta \mid \mathrm{x})$ & $\mathrm{E}(\theta \mid \mathrm{x})$ & $\mathrm{V}(\theta \mid \mathrm{x})$ & $\mathrm{E}(\theta \mid \mathrm{x})$ \\
\hline-0.8 & -.6651 & .0780 & -.7473 & .0102 & -.7868 \\
-0.6 & -.5787 & .0129 & -5930 & .0031 & -.5979 \\
-0.4 & -.3999 & .0033 & -3984 & .0033 & -.3984 \\
-0.2 & -.1992 & .0033 & -.1985 & .0033 & .0032 \\
0.2 & .2022 & .0033 & .2014 & .0033 & .0033 \\
0.4 & .4029 & .0033 & .4012 & .0032 & .2014 \\
0.6 & .5809 & .0136 & .5955 & .0031 & .4012 \\
0.8 & .6751 & .0769 & .7511 & .0099 & .0033 \\
\hline
\end{tabular}




\section{References}

[1] S. Shaarawy, L. Bromeling, Bayesian inferences and forecasts with moving average processes, Communication in Statistics: Theory and Methods 13(15) 91984) 1871-1888.

[2] M.M. Smadi, Bayesian inference of threshold autoregressive moving average models using sample-based methods. PH. D. Dissertation, Colorado State University, Fort Collins, U.S.A. 1997.

[3] M. Smadi, A. Abu-Taleb, On Bayes estimation of first order moving average model, Journal of Mathematics and Statistics 2(3) (2006) 422426.

[4] S. Koreisha, T. Pukkila, 1990, A generalized least-squares approach for estimation of autoregressive moving-average models, Journal of time series, 11(2) (1990) 139-151.

[5] T. Safadi, P.A. Morettin, Bayesian analysis of threshold autoregressive moving average models, Sankhya., 62(3) (2000) $353-371$.

[6] W.S. Cathy, Bayesian inferences and forecasting in bilinear time series models, Communication in Statistics: Theory and Methods, 21(6) (1992) 1725-1743.

[7] M.M. Smadi, M.T. Alodat, Bayesian threshold moving average models, 10(1) 2011 (262-267). 